\title{
An extended goal programming methodology for analysis of a network encompassing multiple objectives and stakeholders
}

\author{
Dylan Jones ${ }^{\mathrm{a}, *}$, Helenice Florentino ${ }^{\mathrm{b}}$, Daniela Cantane ${ }^{\mathrm{b}}$, Rogerio Oliveira ${ }^{\mathrm{b}}$ \\ ${ }^{a}$ Centre for Operational Research and Logistics (CORL), Department of Mathematics, University of Portsmouth, UK \\ ${ }^{\mathrm{b}}$ Department of Bio-Statistics, UNESP, Botucatu, São Paulo, Brazil
}

\section{A R T I C L E I N F O}

\section{Article history:}

Received 11 August 2015

Accepted 17 May 2016

Available online 27 May 2016

\section{Keywords:}

Extended goal programming

Multiple objective programming

Renewable energy

\begin{abstract}
A B S T R A C T
This paper proposes a goal programming methodology to ensure that a mix of balance and optimisation is achieved across a hierarchical decision network. The extended goal programming principle is used for this purpose. A model is constructed that provides consideration of balance and efficiency of multiple objectives and stakeholders at each network node level. A goal programming formulation to provide the decision that best meets the goals of the network is given. The proposed model is controlled by three key parameters that represent the level of non-compensation between objectives, level of non-compensation between stakeholders, and level of centralisation in the network. The methodology is demonstrated on an example pertaining to regional renewable energy generation and the results are discussed. Conclusions are drawn as to the effect of different attitudes towards compensatory behaviour between objectives and stakeholders in the network.
\end{abstract}

(C) 2016 Elsevier B.V. All rights reserved.

\section{Introduction}

The initial goal programming model is proposed by Charnes and Cooper (1961) as a means of modelling the satisficing philosophy of Simon (1957) in a mathematical programming framework. Since then, the technique of goal programming has been developed to encompass many variants and fields of application (Jones \& Tamiz, 2010). The fundamental variants are lexicographic, which combines ordering and satisficing philosophies; weighted, which combines optimising (Pareto, 1896) and satisficing philosophies and Chebyshev, which combines satisficing and balancing (Rawls, 1973) philosophies. More recently advanced variants have been proposed that provide effective frameworks for combining philosophies and modelling modern complex decision problems involving multiple, conflicting goals. These include meta-goal programming (Rodríguez Uría, Caballero, Ruiz, \& Romero, 2002), extended goal programming (Romero, 2001, 2004) and multi-choice goal programming (Chang, 2008). The meta-goal programming model proposes the concept of a meta-goal, a high level goal that goes beyond a single goal and gives an overall measure of satisfaction for the decision maker. In this context the extended goal programming model can be seen as comprising two meta-objectives: the minimisation of the weighted, normalised sum of unwanted deviations from

\footnotetext{
* Corresponding author. Tel.: +44 2392846362.

E-mail address: Dylan.Jones@port.ac.uk (D. Jones).
}

the set of goals (using the $L_{1}$ distance function and representing efficiency and optimising principles) and the minimisation of the maximal weighted, unwanted, normalised deviation from amongst the set of goals (using the $L_{\infty}$ distance function and representing balancing and social justice principles). A parametric analysis can be undertaken to determine the trade-off between balance and optimisation in decision or objective space. On the other hand, the multi-choice goal programming model allows the decision maker to specify multiple target values for each goal. Definitions of the $L_{p}$ distance functions and their relationship within the goal programming model are given in Romero, Tamiz, and Jones (1998). In combination, the above goal programming variants provide a comprehensive methodology for modelling diverse decision maker preferences and underlying philosophies. However, they also have the commonality that they focus on the expressed goals of a single entity, either a single decision maker or a group of decision makers with unified goals. This is a different decision making situation to a network of stakeholders, all of whom have some influence on the decision(s) to be made but may have different preferences and views on importance and compensation amongst the set of objectives under consideration. The methodology presented in this paper is concerned with examining the mix between balancing and optimisation philosophies over a network of stakeholders. The effects of compensatory $\left(L_{1}\right)$ and non-compensatory $\left(L_{\infty}\right)$ behaviour with respect to both the multiple stakeholders in different parts of the network and multiple objectives is examined. Extended goal programming is chosen as the base technique for 
the methodology due to its synergies and similarities with the required analysis. In fact, the methodology developed in this paper can also be seen as an extension of and contribution to the literature on extended goal programming.

The remainder of the paper is divided into 4 sections. Section 2 presents a more detailed discussion of extended goal programming as well as detailing the literature on goal programming for networks of decisions and multiple stakeholders. Section 3 develops the methodology for, and algebraic form of, the network extended goal programming model. Section 4 presents a hypothetical example from the field of renewable energy planning in order to demonstrate the methodology and discusses the results. Finally, Section 5 draws conclusions.

\section{Relevant goal programming topics}

This Section reviews the current state-of-the-art of goal programming in the topics of relevance to this paper. These are divided into three sub-sections. The extended goal programming model variant; the use of goal programming to model networks of decisions and multiple stakeholders; and the inclusion of the Rawlsian philosophies of social justice, balance, and fairness.

\subsection{Extended goal programming}

As described in Section 1, the extended goal programming model is introduced by Romero $(2001,2004)$ to allow a parametric analysis of the trade-off between efficiency and balance between the levels of achievement of the goal target values. Lexicographic and non-lexicographic forms of the model are presented for the cases of the presence and absence of a lexicographic ordering of goals respectively. As this paper is concerned primarily with investigations of efficiency-balance trade-offs between stakeholders and objectives over a decision network rather than prioritising of objectives the non-lexicographic form of the extended goal programming model is used. Assuming a linear form of the achievement function, percentage normalisation, and positive target values $\left(b_{i}>0, i=1, \ldots, q\right)$ (Jones \& Tamiz, 2010) gives the following algebraic model:

Min $a=\alpha \lambda+(1-\alpha)\left\{\sum_{i=1}^{q}\left(\frac{u_{i} n_{i}}{b_{i}}+\frac{v_{i} p_{i}}{b_{i}}\right)\right\}$

Subject to:

$\frac{u_{i} n_{i}}{b_{i}}+\frac{v_{i} p_{i}}{b_{i}} \leq \lambda \quad i=1, \ldots, q$

$f_{i}(\underline{x})+n_{i}-p_{i}=b_{i} \quad i=1, \ldots, q$

$\underline{x} \in F$

$n_{i}, \quad p_{i} \geq 0 \quad i=1, \ldots, q$

Model (1)-(5) is defined as having $q$ objectives and a set $\underline{x}$ of decision variables. $f_{i}(\underline{x})$ is the achieved value of the $i$ th objective which has an associated target value of $b_{i}$. Deviational variables $n_{i}$ and $p_{i}$ denote the negative and positive deviations from the $i$ th target value respectively. The maximal weighted deviation from amongst the set of unwanted deviations is denoted by $\lambda$. The weights $u_{i}$ and $v_{i}$ are associated with the relative level of importance associated with the minimisation of the negative and positive deviational variables from the $i$ th target value respectively. Unwanted deviations are given a positive weight and deviations which are not desired to be minimised are given a zero weight. $\alpha$ is a parameter which controls the relative importance of efficiency and equity in the model. Note that whilst this model has assumed percentage normalisation and positive target values, other forms of normalisation could also be considered, which in turn could allow for the inclusion of non-positive target values.

The extended goal programming formulation allows for the inclusion and combination of the optimisation, balancing, and satisficing underlying philosophies of a single decision making entity (Jones \& Tamiz, 2010). The satisfying philosophy is evident in the set of goals. The optimising philosophy is achieved via the minimisation of the weighted sum of deviations (second term in the achievement function (1)) with the use of sufficiently optimistic target goal values. The balancing philosophy is achieved through the inclusion of the maximal deviation (first) term in the achievement function (1). Furthermore, the balance between optimisation (efficiency) and balance (equity) can be controlled at each priority level through the parameter $\alpha$ which can be varied between complete emphasis on optimisation $(\alpha=0)$ and complete emphasis on balance $(\alpha=1)$. The EGP framework is therefore a comprehensive tool for the inclusion of three types of underlying philosophies amongst a set of objectives on a single decision level into the goal programming framework. This framework has been further enhanced by since its inception. (Jones \& Jimenez, 2013) propose two further meta-objectives to add to the original two of optimisation and balance. These are the number of goals achieved (representing a target achieving philosophy) and consistency with pairwise comparison matrix judgements in the case that they are used to provide the set of weights. It has hitherto been developed in single decision layer form rather than in hierarchical network form, as proposed in this paper.

\subsection{Networks of decisions and multiple stakeholders}

Many multiple criteria problems involve multiple stakeholders, who are defined as entities (organisations, individuals, or societal groups) who are affected by the decision to be made. An indicative, but not exhaustive, review of the use of goal programming for problems with either multiple stakeholders or a network structure is given by the remainder of this paragraph. The initial example of the use of goal programming to make decisions over a hierarchical network is given by Charnes, Haynes, Hazleton, and Ryan (1975) who formulate a model a three level network for environmental land use planning. The model considers economic and environmental objectives, as well as stakeholders including demographical and industrial groups, and has an assumed governmental decision maker. Recent examples of goal programming models that consider multiple stakeholders include Nixon, Dey, Davies, Sagi, and Berry (2014) who consider the optimal location of biomass plants on a regional level in India considering the needs of farmers, investors, and downstream consumers of electricity. Gebrezgabher, Meuwissen, and Oude Lansink (2014) construct an economically, socially and environmentally sustainable manure management system, using a combination of compromise programming and goal programming based AHP that considers the needs of both farmers and the wider society. Giménez, Bertomeu, Diaz-Balteiro, and Romero (2013) apply extended goal programming for Eucalyptus plantation management considering economic and sustainability criteria and give suggestions for extensions to a multiple stakeholder situation. Li, Beullens, Jones, and Tamiz (2008) develop a two-level decision model of a hospital considering bed allocation at both a departmental and hospital level to meet economic and performance goals. The above examples demonstrate that goal programming is indeed a pragmatic tool for considering different stakeholder groups and complex decisions over a network that may represent geographical regions, technological types, multiple organisations or subdivisions of an organisation, socioeconomic groups, or communities. However, there is not yet 
a clear methodological basis as to how the interests of these groups are represented and combined across a decision network, especially when the mix of compensatory and non-compensatory behaviour amongst both stakeholders and objectives is present. This paper makes a contribution towards the development of a relevant methodology in this area.

Note that this paper makes a distinction between multiple stakeholders and multiple decision makers. The latter case assumes the responsibility for a decision lies with a group of decision makers. The methodology in this case belongs to the field of group decision making. A recent example of the use of goal programming to aid group decision making in the context of the Analytical Hierarchy Process is given by Wang and Li (2015). In contrast, this paper is concerned with the case where the actual decision belongs to a single decision maker with responsibility for considering the needs and opinions of multiple stakeholders who will be affected by the decision. It is often the case that a given stakeholder will have more interest in, and hence place more emphasis on, a subset of the objectives and/or decisions to be made. Different stakeholders may hence have different views on the level of importance to be assigned to individual objectives and the level of compensation between objectives and between stakeholders that should be employed.

\subsection{Concepts of social justice, fairness and balance in goal programming}

The concept of fairness or balance is originally introduced into the goal programming framework by Flavell (1976) who proposes the Chebyshev goal programming model. This variant is based around the $L_{\infty}$ distance function which links to the Rawlsian theory of social justice (Rawls, 1973). Minimising the maximum weighted, normalised deviation from a goal ensures that a balance between the levels of satisfaction of the goals is achieved. Chebyshev goal programming is hence associated with the concepts of fairness, equality, and social justice. However, it is important to note that the Chebyshev variant only explicitly treats the fairness and balance between objectives rather than between stakeholders or different subset of objectives that may have importance in the context of the model. The Chebyshev variant is integrated in the variant encompassing extended goal programming model as detailed in Section 3. It has been practically used to control vibration in vehicle suspension (Li, Liang, Wang, \& Dong, 2012); to allocate maintenance technicians to toolsets in a semiconductor manufacturing plant (Ignizio, 2004); and to select portfolios of mutual funds (Tamiz, Azmi, \& Jones, 2013).

\section{Formulation of extended goal programming model with a network of multiple stakeholders and objectives}

This section proposes a model for parametric consideration of efficiency and balance over hierarchical decision network consisting of $L$ layers with multiple objectives and multiple stakeholders. It is assumed that each stakeholder is associated with one particular node in the network which could, for instance, represent a particular geographical region or sub-division of an organisation. The presented model does not however preclude extension to cover stakeholders with interests that cover multiple nodes or layers of the network. A stakeholder will have their own preferential data with respect to the set of objectives regarded as important, and in some cases place minimal or no importance on a particular objective. Each stakeholder may also have different views on the level of compensation between objectives. These preferential considerations are incorporated into the model presented in formulation (6)-(11). Each network layer $l$ consists of $J^{l}$ nodes. The following algebraic model has the capacity to consider balance and efficiency both amongst objectives at a given node and amongst stakeholders at a given level:

$$
\begin{aligned}
\text { Min } a=w_{1}[ & \left.\alpha^{j^{1}} \lambda^{j^{1}}+\left(1-\alpha^{j^{1}}\right) \sum_{k=1}^{K}\left(\frac{u_{k}^{j^{1}} n_{k}^{j^{1}}}{b_{k}^{j^{1}}}+\frac{v_{k}^{j^{1}} p_{k}^{j^{1}}}{b_{k}^{j^{1}}}\right)\right] \\
& +\sum_{l=2}^{L} w_{l}\left[\beta_{l} D_{l}+\left(1-\beta_{l}\right)\right. \\
& \left.\times \sum_{j^{l}=1}^{J^{l}}\left\{\alpha^{j^{l}} \lambda^{j^{l}}+\left(1-\alpha^{j^{l}}\right) \sum_{k=1}^{K}\left(\frac{u_{k}^{j^{l}} n_{k}^{j^{l}}}{b_{k}^{j^{l}}}+\frac{v_{k}^{j^{l}} p_{k}^{j^{l}}}{b_{k}^{j^{l}}}\right)\right\}\right]
\end{aligned}
$$

Subject to,

$$
f_{k}^{j^{l}}(\underline{x})+n_{k}^{j^{l}}-p_{k}^{j^{l}}=b_{k}^{j^{l}} \quad k=1, \ldots, K ; \quad j^{l}=1, \ldots, J^{l} ; \quad l=1, \ldots, L
$$

$$
\begin{aligned}
& \frac{u_{k}^{j^{l}} n_{k}^{j^{l}}}{b_{k}^{j^{l}}}+\frac{v_{k}^{j^{l}} p_{k}^{j^{l}}}{b_{k}^{j^{l}}} \leq \lambda^{j^{l}} \quad k=1, \ldots, K ; \quad j^{l}=1, \ldots, J^{l} ; \quad l=1, \ldots, L \\
& \left\{\alpha^{j^{l}} \lambda^{j^{l}}+\left(1-\alpha^{j^{l}}\right) \sum_{k=1}^{K}\left(\frac{u_{k}^{j^{l}} n_{k}^{j^{l}}}{b_{k}^{j^{l}}}+\frac{v_{k}^{j^{l}} p_{k}^{j^{l}}}{b_{k}^{j^{l}}}\right)\right\} \leq D_{l} \\
& j^{l}=1, \ldots, J^{l} ; \quad l=1, \ldots, L \\
& \underline{x} \in F \\
& n_{k}^{j^{l}}, \quad p_{k}^{j^{l}} \geq 0 \quad k=1, \ldots, K ; \quad j^{l}=1, \ldots, J^{l} ; \quad l=1, \ldots, L \\
& \lambda^{j^{l}} \geq 0 \quad j^{l}=1, \ldots, J^{l} ; \quad l=1, \ldots, L ; \quad D_{l} \geq 0 \quad l=1, \ldots, L \quad(11
\end{aligned}
$$

where $f_{k}^{j^{l}}(\underline{x})$ is a function of decision variable set $\underline{x}$ that gives the achieved value of objective $k$ at node $j^{l}$ at network level $l . n_{k}^{j^{l}}$ and $p_{k}^{j^{l}}$ are the negative and positive deviations from goal target level $b_{k}^{j^{l}}$ of objective $k$ at node $j^{l}$ at network level $l$ respectively. $u_{k}^{j^{l}}$ and $v_{k}^{j^{l}}$ are the weights associated with penalisation of negative and positive deviations from the goal target level of objective $k$ at node $j^{l}$ at network level $l$ respectively. If a deviation is not to be penalised then its associated weight should be set to zero. If a particular objective is not relevant at a node then both associated weights should be set to zero. $\lambda^{j}{ }^{l}$ represents the maximal weighted, normalised deviation from amongst the set of objectives at node $j^{l}$ at network level $l$. $D_{l}$ represents the maximum combined measure of stakeholder dissatisfaction amongst the set of nodes at network level $l$. These are the two key measures of balance in the model. It is also important to note that the first level has been modelled as a separate term in the achievement function (6). This is due to the fact that it represents the centralisation portion of the network as opposed to the other levels, which represent the devolved decision making in the network. Hence there are important philosophical and modelling reasons that justify its separate consideration. A diagrammatical illustration of the extended network goal programming algebraic model (7)-(11) is given in Fig. 1. There are three principal parameter sets in the model:

(1) $w_{l}$ is the relative level of importance given to network level $l$. The set of network level weights $\underline{w}=w_{1}, w_{2}, \ldots, w_{L}$ gives the centralisation versus decentralisation strategy of the decision maker. It is suggested that the network level weights $\underline{w}$ are normalised via the following equation:

$$
\sum_{l=1}^{L} w_{l}=1
$$




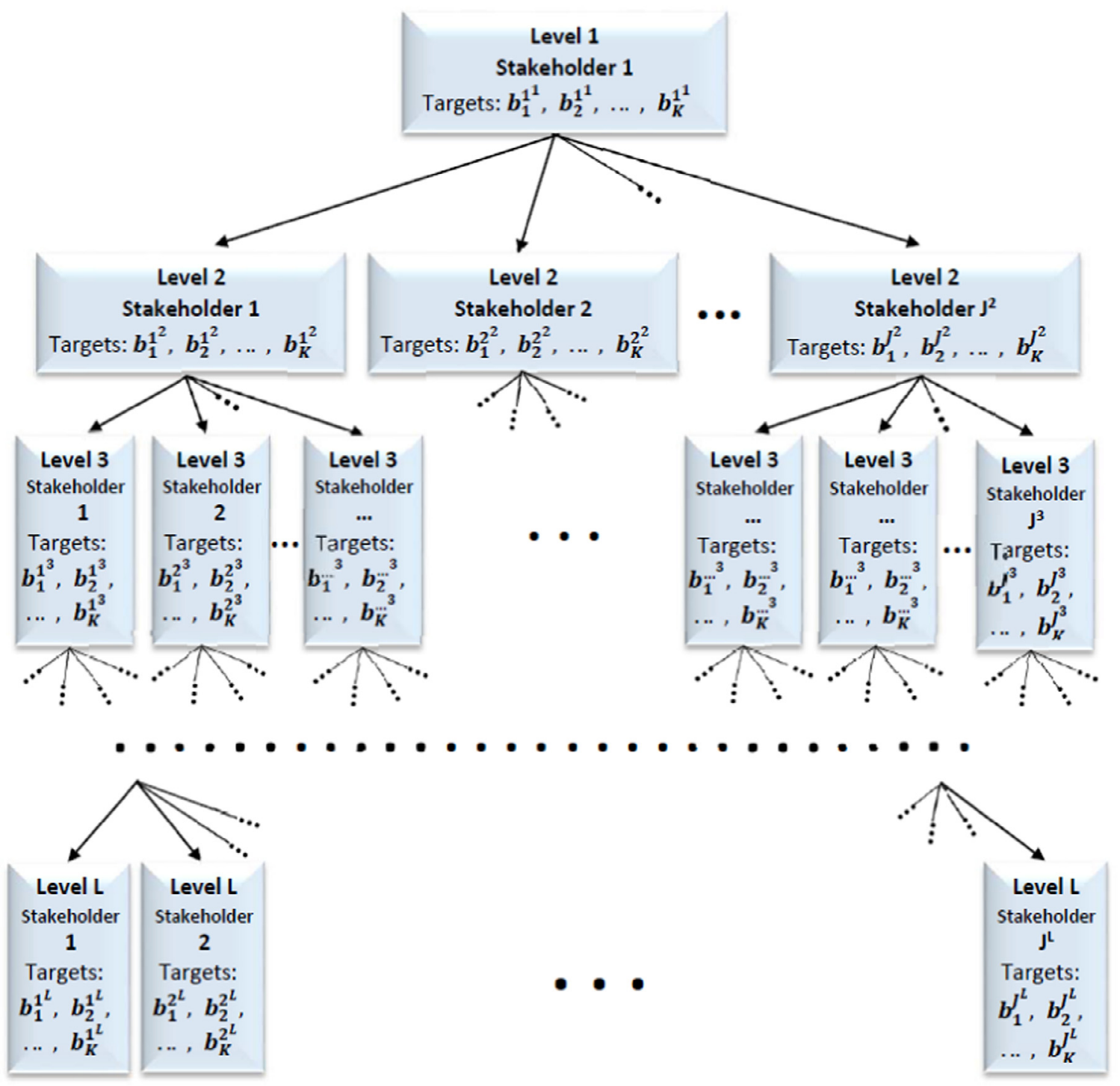

Fig. 1. Diagrammatic illustration of the extended network goal programming model.

(2) $\alpha^{j^{l}}$ gives the level of consideration of balance versus optimisation amongst objectives at node $j^{l}$ at network level $l$. It is subject to the bounds $0 \leq \alpha^{j^{l}} \leq 1$, where $\alpha^{j^{l}}=0$ indicates the stakeholder(s) associated with that node is solely interested in the (weighted sum) efficiency of the objectives and $\alpha^{j^{l}}=1$ indicates the stakeholder(s) associated with that node is solely interested in the (minmax) balance of the objectives. Thus $\alpha^{j^{l}}$ can be seen as a measure of consideration of the balance and efficiency mix between objectives.

(3) $\beta_{l}$ gives the level of consideration of balance versus optimisation amongst stakeholders scores at network level $l$. It is subject to the bounds $0 \leq \beta_{l} \leq 1$, where $\beta_{l}=0$ indicates that importance at network level $l$ is solely given to the average stakeholder dissatisfaction and $\beta_{l}=1$ indicates that importance is solely given to the maximal stakeholder dissatisfaction at that level. Thus $\beta_{l}$ can be seen as a measure of consideration of the balance and efficiency mix between stakeholders at network level $l$.

As previous stated, the decision network is assumed to be controlled by a single decision maker whose role is to consider all stakeholders in the decisions to be made. A parametric analysis around the three key parameter sets is proposed in order that the decision maker gain understanding about the nature of the tradeoffs between balance and efficiency and the effect of compensatory behaviour in the model. An example of a parametric analysis is given in the model presented in Section 4.

\section{An illustrative example}

This section develops an example decision network related to regional development of renewable energy sources in order to test and illustrate the model developed in Section 3. The model has two levels (regional and global) and four sets of goals relating to energy generation, cost, environmental impact, and number of projects developed. Parametric analysis is then performed on a specific four region instance. The data used is hypothetical as the purpose is illustration of method. The objectives and problem formulation are however, inspired by the authors work on various European Union and São Paulo state, Brazil funded projects on the development of mathematical models for renewable energy. 


\subsection{Notation}

Following mathematical programming convention, the model description is divided into required input data; parameters to control the experimentation (the weights are considered as parameters in this instance as, although the decision maker may have an initial estimate, some form of informal or formal sensitivity analysis may be necessary; Jones, 2011); decision variables; and an algebraic model.

\subsubsection{Data \\ $n$ \\ $J$ \\ K \\ $Q_{j}$ \\ $Q_{k k}\left(=Q_{j} \cap Q_{k}\right)$}

$E_{i}$

$E G$

$E R_{j}$

$C_{i}$

$C G$

$C R_{j}$

$H_{i}$

$H G$

$H R_{j}$

$T G_{k}$

$T R_{j k}$

$s_{j}^{\min }$

\subsubsection{Parameters}

$w \quad$ controls global-regional weighting;

$\alpha^{G} \quad$ controls mix of optimisation and balance at a global level;

$\alpha_{j}^{R} \quad$ controls mix of optimisation and balance in individual region $j$;

$\beta \quad$ controls mix of optimisation and balance when considering set of regions;

$u_{E}^{G} \quad$ weight associated with penalising negative deviation from global energy target;

$v_{C}^{G} \quad$ weight associated with penalising positive deviation from global cost target;

$v_{H}^{G} \quad$ weight associated with penalising positive deviation from global environmental target;

$u_{T k}^{G} \quad$ weight associated with penalising negative deviation from global target for electricity generation type $k$;

$u_{E j}^{R} \quad$ weight associated with penalising negative deviation from energy target of region $j$;

$v_{C j}^{R} \quad$ weight associated with penalising positive deviation from cost target of region $j$;

$v_{H j}^{R} \quad$ weight associated with penalising positive deviation from environmental target of region $j$;
$v_{T j k}^{R}$

weight associated with penalising negative deviation from target for electricity generation type $k$ in region $j$.

4.1.3. Decision variables

The following sets of decision and deviation variables are specified

$s_{i}=\left\{\begin{array}{l}1 \text { If project } i \text { selected } \\ 0 \text { otherwise }\end{array} i=1, \ldots, n\right.$

$\lambda^{G}$ maximal deviation from set of global normalised,

$\lambda_{j} \quad$ weighted goals;

$\lambda_{j}^{R} \quad$ maximal deviation from set of global normalised, weighted goals in region $j$;

D maximal measure from amongst the set of regions (the worst performing region);

$n_{E}^{G} \quad$ cegative deviation from global energy target;

$p_{E}^{G} \quad$ positive deviation from global energy target;

$n_{C}^{G} \quad$ negative deviation from global cost target;

$p_{C}^{G} \quad$ positive deviation from global cost target;

$n_{H}^{G} \quad$ negative deviation from global environmental target;

$p_{H}^{G} \quad$ positive deviation from global environmental target;

$n_{T k}^{G} \quad$ negative deviation from global electricity generation type

$k$ target;

$p_{T k}^{G} \quad$ positive deviation from global electricity generation type

$k$ target;

$n_{E j}^{R} \quad$ negative deviation from energy target for region $j$;

$p_{E j}^{R} \quad$ positive deviation from energy target for region $j$;

$n_{C j}^{R} \quad$ negative deviation from cost target for region $j$;

$p_{C j}^{R} \quad$ positive deviation from cost target for region $j$;

$n_{H j}^{R} \quad$ negative deviation from environmental target for region $j$;

$p_{H j}^{R} \quad$ positive deviation from environmental target for region j;

$n_{T j k}^{R} \quad$ negative deviation from electricity generation type $k$ tar-

get for region $j$;

$p_{T j k}^{R} \quad$ positive deviation from electricity generation type $k$ target for region $j$.

4.1.4. Algebraic model

The algebraic form of the two-layer network extended goal programming is given by Eqs. (12)-(26).

$$
\begin{aligned}
\text { Min } a=w[ & {\left[\alpha^{G} \lambda^{G}+\left(1-\alpha^{G}\right)\left(\frac{u_{E}^{G} n_{E}^{G}}{E G}+\frac{v_{C}^{G} p_{C}^{G}}{C G}+\frac{v_{D}^{G} p_{D}^{G}}{H G}\right.\right.} \\
& \left.\left.+\sum_{k=1}^{K} \frac{u_{T k}^{G} n_{T k}^{G}}{T G_{k}}\right)\right] \\
& +(1-w)\left[\beta D+(1-\beta) \sum_{j=1}^{J} \alpha_{j}^{R} \lambda_{j}^{R}+\left(1-\alpha_{j}^{R}\right)\left(\frac{u_{E j}^{R} n_{E j}^{R}}{E R_{j}}\right.\right. \\
& \left.\left.+\frac{v_{C j}^{R} p_{C j}^{R}}{C R_{j}}+\frac{v_{H j}^{R} p_{H j}^{R}}{H R_{j}}+\sum_{k=1}^{K} \frac{v_{T j k}^{R} n_{T j k}^{R}}{T R_{j k}}\right)\right]
\end{aligned}
$$

Subject to,

$$
\begin{aligned}
& \sum_{i=1}^{n} E_{i} s_{i}+\underline{n_{E}^{G}}-p_{E}^{G}=E G \\
& \sum_{i=1}^{n} C_{i} s_{i}+n_{C}^{G}-\underline{p_{C}^{G}}=C G \\
& \sum_{i=1}^{n} H_{i} s_{i}+n_{D}^{G}-\underline{p_{D}^{G}}=H G
\end{aligned}
$$




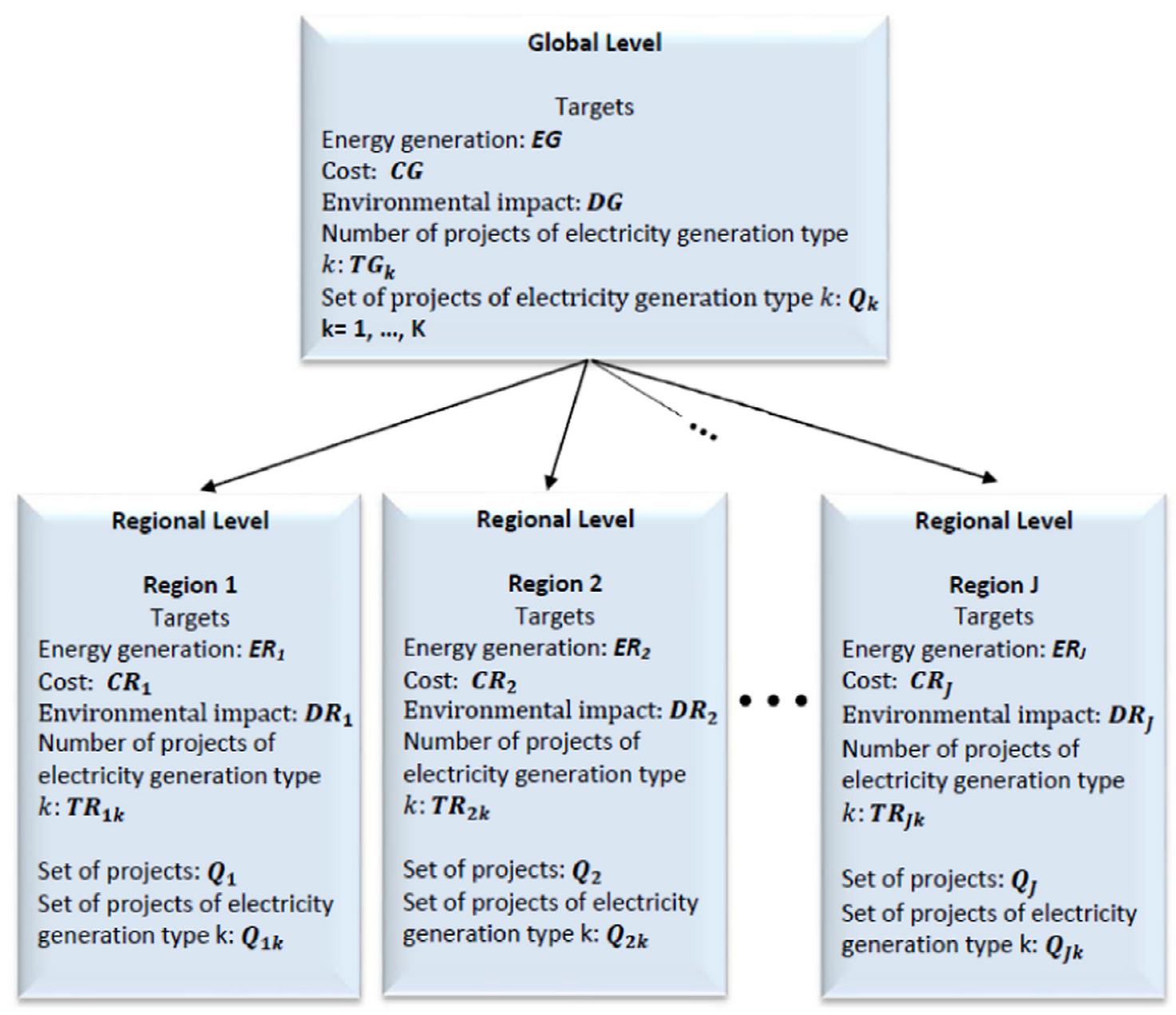

Fig. 2. Diagrammatic illustration of the renewable energy planning example.

$\sum_{i \in Q_{k}} s_{i}+\underline{n_{T k}^{G}}-p_{T k}^{G}=T G_{k} \quad k=1, \ldots, K$

$\sum_{i \in Q_{j}} E_{i} s_{i}+\underline{n_{E j}^{R}}-p_{E j}^{R}=E R_{j} \quad j=1, \ldots, J$

$\sum_{i \in Q_{j}} C_{i} s_{i}+n_{C j}^{R}-\underline{p_{C j}^{R}}=C R_{j} \quad j=1, \ldots, J$

$\sum_{i \in Q_{j}} H_{i} s_{i}+n_{H j}^{R}-p_{H j}^{R}=H R_{j} \quad j=1, \ldots, J$

$\sum_{i \in Q_{j k}} s_{i}+\underline{n_{T j k}^{R}}-p_{T j k}^{R}=T R_{j k} \quad k=1, \ldots, K ; \quad j=1, \ldots, J$

$\frac{u_{E}^{G} n_{E}^{G}}{E G} \leq \lambda^{G}, \frac{v_{C}^{G} p_{C}^{G}}{C G} \leq \lambda^{G}, \frac{v_{H}^{G} p_{H}^{G}}{H G} \leq \lambda^{G}, \sum_{k=1}^{K} \frac{u_{T k}^{G} n_{T k}^{G}}{T G_{k}} \leq \lambda^{G}$

$$
\begin{aligned}
\frac{u_{E j}^{R} n_{E j}^{R}}{E R_{j}} & \leq \lambda_{j}^{R}, \quad \frac{v_{c j}^{R} p_{C j}^{R}}{C R_{j}} \leq \lambda_{j}^{R}, \frac{v_{H j}^{R} p_{H j}^{R}}{H R_{j}} \leq \lambda_{j}^{R}, \sum_{k=1}^{K} \frac{v_{T j k}^{R} n_{T j k}^{R}}{T R_{j k}} \leq \lambda_{j}^{R} \\
& j=1, \ldots, J
\end{aligned}
$$

$$
\begin{aligned}
& \alpha_{j}^{R} \lambda_{j}^{R}+\left(1-\alpha_{j}^{R}\right)\left(\frac{u_{E j}^{R} n_{E j}^{R}}{E R_{j}}+\frac{v_{C j}^{R} p_{C j}^{R}}{C R_{j}}+\frac{v_{H j}^{R} p_{H j}^{R}}{H R_{j}}+\sum_{k=1}^{K} \frac{v_{T j k}^{R} n_{T j k}^{R}}{T R_{j k}}\right) \leq D \\
& j=1, \ldots, J \\
& \sum_{i \in Q_{j}} s_{i} \geq s_{j}^{\min } \quad j=1, \ldots, J \\
& s_{i}=0 \text { or } 1 i=1, \ldots, n ; \quad n_{E}^{G}, p_{E}^{G}, n_{C}^{G}, p_{C}^{G}, n_{H}^{G}, p_{H}^{G}, \lambda^{G} \geq 0 ; n_{T k}^{G}, \\
& p_{T k}^{G} \geq 0 \quad k=1, \ldots, K \\
& j=1, \ldots J \quad k=1 \ldots K
\end{aligned}
$$

where Eq. (12) gives the achievement function to be minimised which is a specific form of achievement function (6). The parameters and decision variables in the second term have one less index that those of (6) as the network has two levels rather than the generic $L$ level case and hence the second term pertains solely to a single (second) network layer. The unwanted deviational variables in Eqs. (13)-(20) are underlined, these are hence the set of unwanted deviational variables to be minimised by achievement function (12). Eqs. (13)-(15) give the global level goals for energy generation, annual cost, and environmental impact respectively. 
Table 1

Project data for specific instance.

\begin{tabular}{llllll}
\hline Project $\left(s_{i}\right)$ & Region & Type & Energy $\left(E_{i}\right)$ & Cost $\left(C_{i}\right)$ & Environmental Impact $\left(H_{i}\right)$ \\
\hline 1 & 1 & 1 & 34 & 12 & 5 \\
2 & 1 & 2 & 23 & 15 & 6 \\
3 & 1 & 1 & 24 & 18 & 7 \\
4 & 2 & 3 & 25 & 19 & 9 \\
5 & 2 & 1 & 56 & 45 & 3 \\
6 & 2 & 2 & 12 & 12 & 4 \\
7 & 3 & 2 & 14 & 34 & 6 \\
8 & 3 & 3 & 19 & 31 & 9 \\
9 & 3 & 2 & 72 & 64 & 2 \\
10 & 3 & 3 & 54 & 43 & 4 \\
11 & 4 & 3 & 12 & 14 & 7 \\
12 & 4 & 3 & 96 & 85 & 8 \\
13 & 4 & 1 & 54 & 45 & 9
\end{tabular}

Equation set (16) gives the goals for the global number of projects of the $K$ different electricity generation types selected. Equation sets (17)-(19) give the $J$ regional level goals for energy generation, annual cost, and environmental impact respectively. Equation set (20) gives the $J$ regional level goals for the global number of projects of the $K$ different electricity generation types selected. Inequality set (21) ensures that the weighted, normalised, unwanted deviation from each global goal target is less than or equal to the maximal global value $\left(\lambda^{G}\right)$. Inequality set (22) ensures that for each region $j$ the weighted, normalised, unwanted deviation from each goal target is less than or equal to the maximal value for that region $\left(\lambda_{j}^{R}\right)$. Note that at both a global and at a regional level the deviations from the target numbers of projects to be funded are considered as a set rather than separately by technology type. This is to avoid over-emphasis being placed on this set of goals when calculating the maximal deviation. Inequality set (23) ensures that each region's composite score (i.e. the parametric combination of the worst case and average deviations) is less than or equal to the worst case regional score $(D)$. Inequality set (24) ensures that at least the minimal number of projects is selected in each region. Eqs. (25) and (26) give the set of sign restrictions for deviation variables in the model. It is noted that this model is a mixed binary problem that can be solved by an integer programming solution algorithm. The number of binary variables is equivalent to the number of potential projects $(n)$. A diagrammatic illustration of algebraic model (12)-(26) is given in Fig. 2.

\subsection{Experimentation on specific instance}

In order to demonstrate the effects of the level of compensatory behaviour between objectives and between stakeholders and the level of centralisation in decision making, a specific four region instance of model (12)-(26) is constructed. The data giving region, type, energy output, cost and environmental impact of each project for this instance is given in Table 1. Furthermore, the global and regional targets and minimal projects per region are set as follows:

$$
\begin{aligned}
E G & =350 ; \quad C G=60 ; \quad H G=15 ; \quad T G_{k}=4, k=1, \ldots, 3 ; \\
E R_{j} & =80, \quad j=1, \ldots, 4 ; \\
C R_{j} & =25, \quad j=1, \ldots, 4 ; \quad H R_{j}=5, \quad j=1, \ldots, 4 ; \quad s_{j}^{\min }=1, \\
j & =1, \ldots, 4 ; \\
T R_{j k} & =1, \quad j=1, \ldots, 4, \quad k=1, \ldots, 3 .
\end{aligned}
$$

An experimental analysis with respect to the three key parameters $w, \alpha$, and $\beta$ is conducted. In order to control the number of executions, the assumption that the level of compensatory behaviour between objectives remains constant throughout the network is made. That is, $\alpha=\alpha^{G}=\alpha_{j}^{R} \forall j$. This is a reasonable assumption in the context of energy planning, where no regional

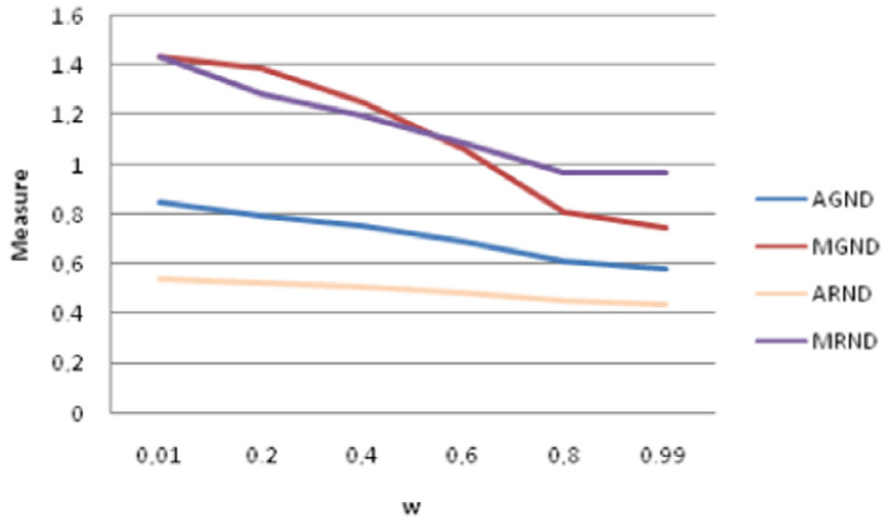

Fig. 3. Effect of variance of parameter $w$ on set of measures.

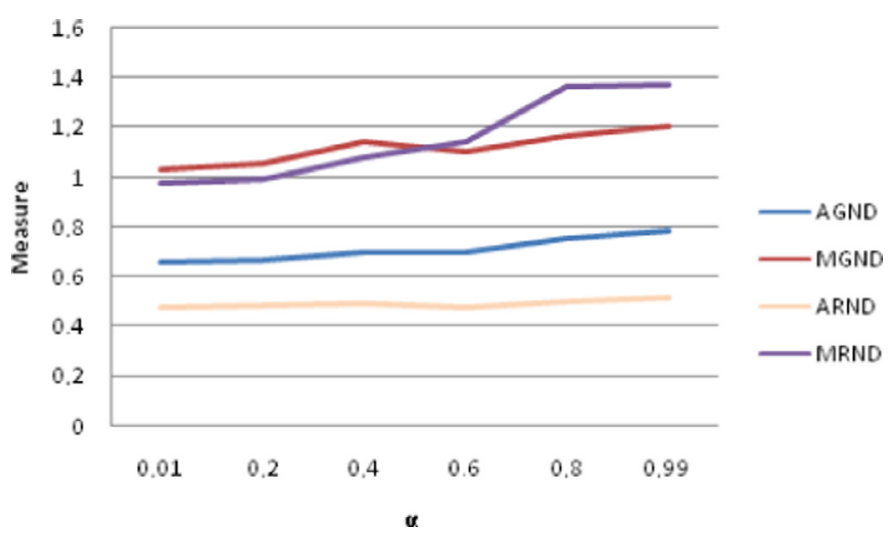

Fig. 4. Effect of variance of parameter $\alpha$ on set of measures.

or central stakeholder would wish to be seen as more or less compensatory in its approach than others, leading to a settling down around a common level of tolerance of compensation. These considerations give a three-parameter model, each of which is discretised into six points on its zero to one range $(0.01,0.2,0.4,0.6$, $0.8,0.99$ ). Note that the values of 0.01 and 0.99 have been used as the end points rather than 0 and 1 in order to avoid potential Pareto inefficiency at the meta-objective level. This leads to 216 optimisations, for which the average and maximal deviations at central and regional level are measured. This is in order to judge whether the parameters giving emphasis on centralisation, optimisation, and balance are working effectively and to draw conclusions about their effects. As the min purpose is to investigate the effect of varying $w, \alpha$, and $\beta$, an equal weight solution is used in order to ensure that all stakeholders and objectives are equally considered. The models are solved via the LINGO software on a PC machine with $3.10 \mathrm{GHz}$ processor speed and 4GB RAM, with all models solving in less than the minimal recording time of one second.

\subsubsection{Results}

Figs.3-5 show the effects of varying the single parameters $w, \alpha$, and $\beta$ respectively, with each observation compromising of the mean of the relevant measure over the 36 values of the other two parameters. The four measures used are:

- AGND: Average global normalised deviation:
$\left(\frac{n_{E}^{G}}{E G}+\frac{p_{C}^{G}}{C G}+\frac{p_{H}^{G}}{H G}+\sum_{k=1}^{3} \frac{n_{T k}^{G}}{T G_{k}}\right) / 4$.

- MGND: Maximum global weighted normalised deviation: $\operatorname{Max}\left(\frac{n_{E}^{G}}{E G}, \frac{p_{C}^{G}}{C G}, \frac{p_{H}^{G}}{H G}, \sum_{k=1}^{3} \frac{n_{T k}^{G}}{T G_{k}}\right)$. 


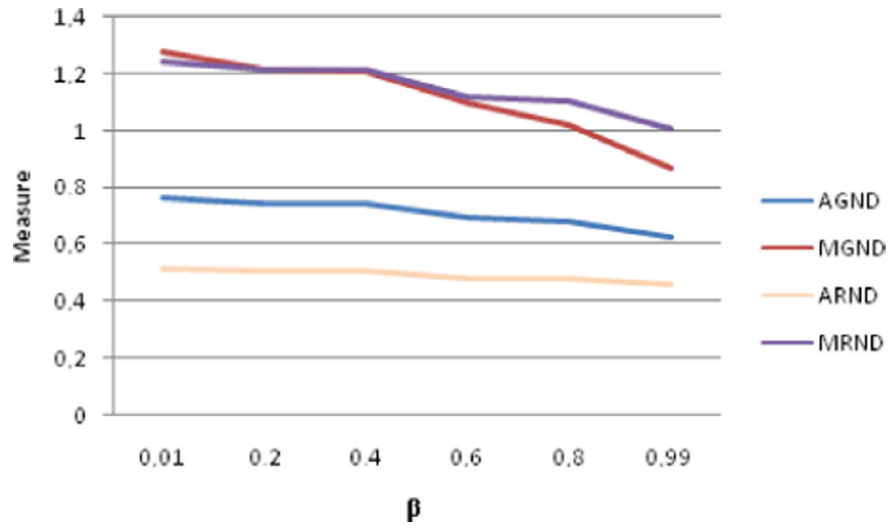

Fig. 5. Effect of variance of parameter $\beta$ on set of measures.

- ARND: Average regional normalised deviation: $\sum_{j=1}^{4}\left(\frac{n_{E j}^{R}}{E G_{j}}+\frac{p_{C j}^{R}}{C G_{j}}+\frac{p_{H j}^{R}}{H G_{j}}+\sum_{k=1}^{3} \frac{n_{T j k}^{R}}{T G j_{k}}\right) / 16$.

- MRND: Maximum regional normalised deviation: $\operatorname{Max}\left(\frac{n_{E j}^{R}}{E G_{j}}, \frac{p_{C j}^{R}}{C G_{j}}, \frac{p_{H j}^{R}}{H G_{j}}, \sum_{k=1}^{3} \frac{n_{T j k}^{R}}{T G j_{k}}, \quad j=1, \ldots, 4\right)$.

Figs. 6-8 give the effects of varying two of the three parameters within their defined range values. The same four measures as in Figs. 3-5 (AGND, MGND, ARND, MRND) are used. Each observation is the mean of the six values of the third parameter.
Table 2

Key solutions in meta-objective and decision space.

\begin{tabular}{llllllll}
\hline$w$ & Alpha & Beta & AGND & MGND & ARND & MRND & Projects funded \\
\hline 0.2 & 0.2 & 0.2 & 0.794 & 1.400 & 0.528 & 1.200 & $1,2,4,8,11$ \\
0.8 & 0.2 & 0.2 & 0.566 & 0.780 & 0.447 & 0.850 & $1,6,8,11$ \\
0.2 & 0.2 & 0.8 & 0.794 & 1.400 & 0.528 & 1.200 & $1,2,4,8,11$ \\
0.8 & 0.2 & 0.8 & 0.565 & 0.780 & 0.447 & 0.850 & $1,6,8,11$ \\
0.4 & 0.4 & 0.4 & 0.794 & 1.400 & 0.528 & 1.200 & $1,2,4,8,11$ \\
0.6 & 0.6 & 0.6 & 0.794 & 1.400 & 0.528 & 1.200 & $1,2,4,8,11$ \\
0.2 & 0.8 & 0.2 & 0.882 & 1.667 & 0.562 & 1.600 & $1,2,4,6,8,11$ \\
0.8 & 0.8 & 0.2 & 0.633 & 0.733 & 0.440 & 1.200 & $1,2,6,10,11$ \\
0.2 & 0.8 & 0.8 & 0.882 & 1.667 & 0.562 & 1.600 & $1,2,4,6,8,11$ \\
0.8 & 0.8 & 0.8 & 0.633 & 0.733 & 0.440 & 1.200 & $1,2,6,10,11$ \\
\hline
\end{tabular}

Figs. 3-8 have focussed on the visualisation of the solution in parameter space, considering a set of measures that are essentially meta-objectives, that is they are functions of a number of unwanted deviation variables (Jones \& Jimenez, 2013). This is in line with the focus of this paper which is that of parametric analysis of the meta-objective space. In order to understand the solution in the decision space (Jones, 2011), Table 2 presents the solutions found at the eight corner points of the parameter space and two central points. Columns 1-3 give the solution in parameter space, columns 4-7 give the solution in the four dimensional meta-objective space, and column 8 gives the solution in decision space in terms of the set of funded projects.

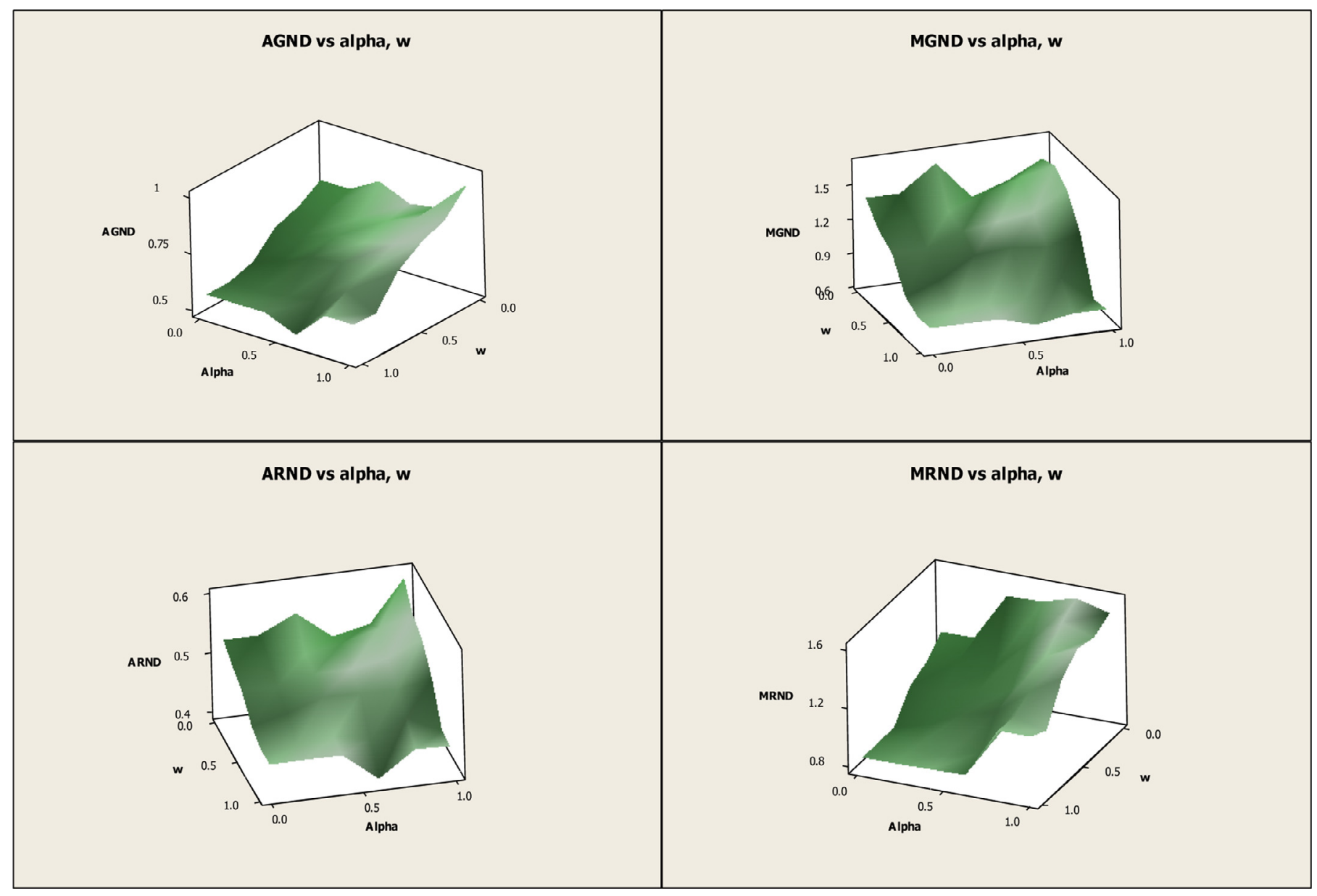

Fig. 6. Effect of variance of parameters $w, \alpha$ on set of measures. 


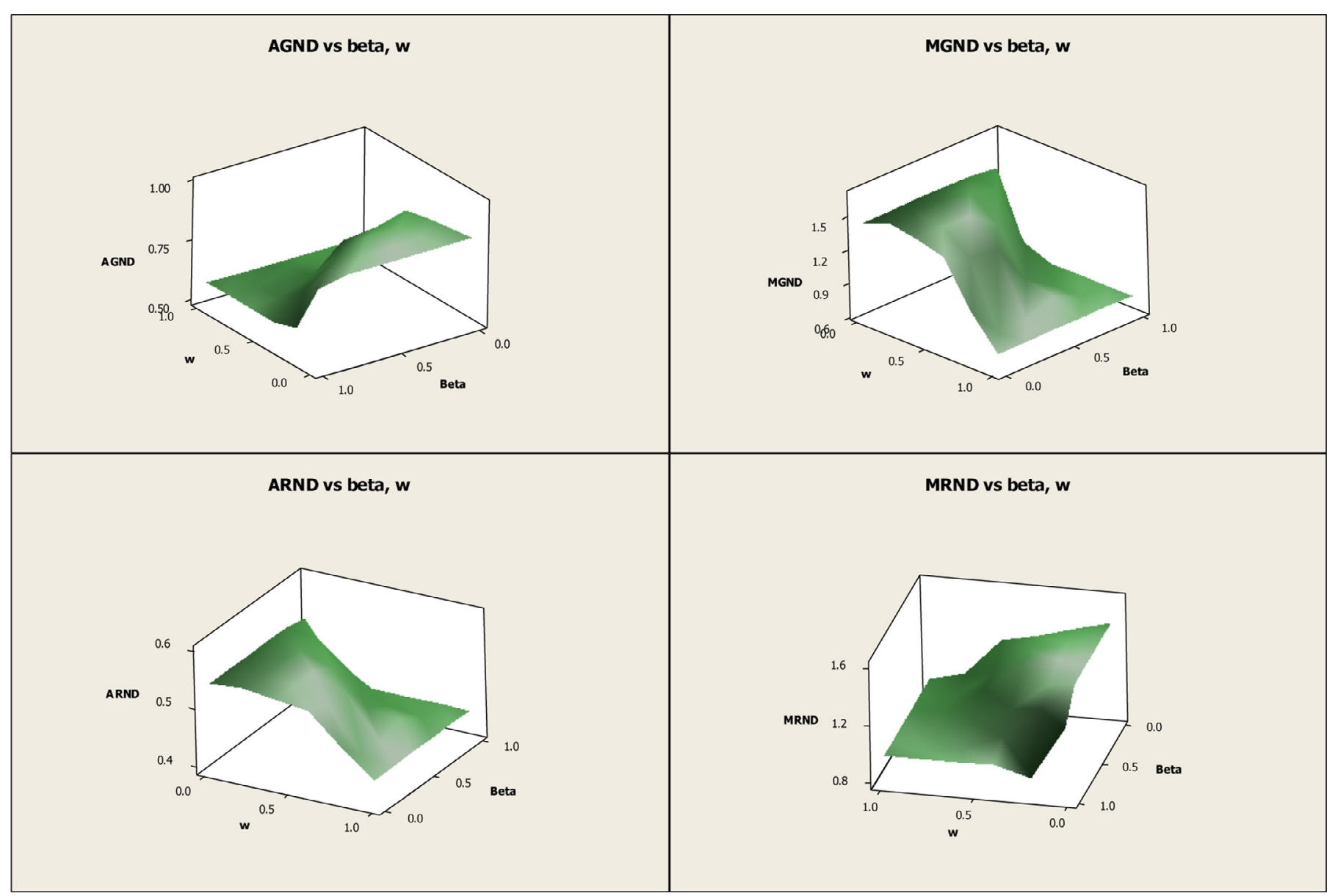

Fig. 7. Effect of variance of parameters $w, \beta$ on set of measures.

\subsubsection{Discussion of results}

Considering the first order effects, an increase in parameter $w$ (Fig. 3) implies a growing level of centralisation of decision making, as the central decision maker retains a greater proportion of the total weight and passes down a lesser proportion to the lower network level. In the example, this is shown to have the effect of reducing (i.e. improving) all of the four measures, thus showing an advantage not only on the global level but also on the regional level. This shows that a level of benevolent coordination is of value in decision networks such as the one proposed in this paper. However, it is also noted that the level of improvement is more pronounced on the global than on the regional level. This shows that whilst centralisation of decision making may have symbiotic advantage for the whole network, the maximum level of improvement in the example is found at the central level. An increase in parameter $\alpha$ (Fig. 4) implies an increase in the level of noncompensation amongst objectives for all stakeholders across the network. That is, stakeholders are less willing to accept a worsening in the achieved value of one objective in order to gain an improved value in another objective. Fig. 4 demonstrates that increasing the level of compensation amongst objectives in the example leads to worse values for all four measures. This indicates that if stakeholders were to allow for more flexibility in terms of trade-offs between objectives, then a better overall solution on both a global and regional scale could be achieved. An increase in the parameter $\beta$ (Fig. 5) implies an increase in the level of noncompensation between stakeholders at different nodes in the network. That is, stakeholders are less willing to accept a worsening in their position in order that the overall position is improved.
Fig. 5 shows that increasing the level of non-compensation between stakeholders in the example leads to an improvement in all of the four measures. This shows that in order to build an effective consensus in the example, it is beneficial to ensure that all stakeholders are effectively represented and not overly disadvantaged to the gain of others.

A common feature of all of the three single parameter effects (Figs. 3-5) is that the sensitivity to parameter change is higher for the two maximal measures than for the two average measures. This indicates that determining the correct parametric mix or conducting sufficient parametric sensitivity analysis is important in ensuring that the "worst off" stakeholders in any decision are not overly disadvantaged. This will help to promote the building of a consensus amongst stakeholders.

Considering the two parameter effects, Figs. 6-8 confirm the conclusions drawn above from the single parameter effects. It can be seen that it most cases there is a general trend of increase in both of the parameter directions identified in the analysis of Figs. 3-5. This trend is more pronounced in the two maximal measures, MGND and MGRD. It is noted, however that there are some extreme parameter values which negate the sensitivity of the other parameter. See for instance the $w=0.99$ value of all four measures in the $w, \beta$ parametric analysis of Fig. 7 . There is no sensitivity towards the parameter $\beta$ at this level. This is an indication of the fact that if an extreme degree of centralisation is used, the sensitivity between stakeholders is lost. Some of the two parameter analyses also demonstrate areas of stability in parameter space. For instance, the MRND graph of the $w, \alpha$ analysis shows there is an area of low $\alpha$ and high $w$ that will lead to good values of this 


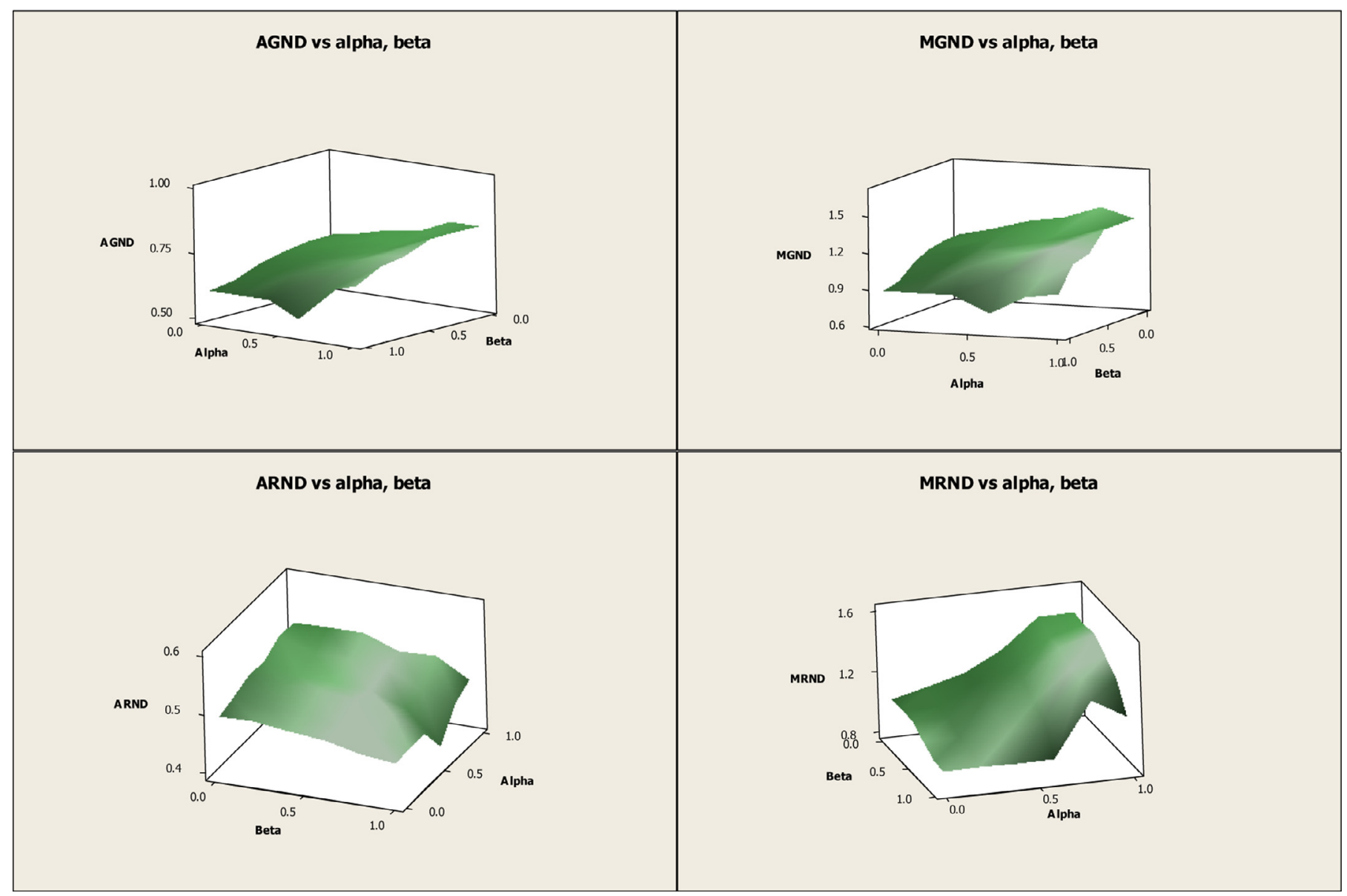

Fig. 8. Effect of variance of parameters $\alpha, \beta$ on set of measures.

measure. This indicates a region with a high level of centralisation and low level of non-compensation between objectives where good values of regional balance can be found.

With respect to the analysis of the solution in decision space given in Table 2, it can be seen that some solutions are repeated, again indicating a lack of sensitivity to parameter change in some, relatively limited regions in the parameter space. The trade-offs taking place in the meta-objective space across the entire region are evident from the values in the four meta-objective columns. The last column demonstrates that, for this example, these changes are achieved by relatively small changes in decision space, with the addition or removal of marginal projects to a set of core projects that appear in all solutions. It is also recognised that some of the maximal deviations are significantly high, rising to a maximum deviation of 1.667 beyond the goal value. This is mainly driven by the deliberate setting of challenging goal values in order to ensure Pareto inefficiency does not occur (Jones \& Tamiz, 2010), but also results the challenge of satisfying all stake-holders across a multiobjective network.

\section{Conclusions}

This paper has extended the methodology of extended goal programming to consider a decision network containing multiple stakeholders and objectives. The motivation for doing so was the occurrence of situations that require this type of coordinated network goal programme in the authors work on renewable energy. This should be regarded as an extension and enhancement of the goal programming paradigm to encompass the type of decision problems with conflicting objectives across a network of stakeholders that are now arising in modern applications. For instance develop a single-layer extended goal programme for offshore wind farm location that would benefit from extension to a decision making network if greater number and types of renewable energy projects were to be included. A demonstrative example from the renewable energy sector has been formulated in Section 4 and its results analysed. Although the model pertains to renewable energy, the methodology presented in Sections 2 and 3 is generic and hence the results could be applied to any decision network with multiple objectives and stakeholders. Potential examples could include transportation networks, computer networks, and decision making in large hierarchical organisations including defence organisations, universities, and health service providers.

The inclusion of the numerical example in Section 4 is intended as a demonstrative concept. The presented results show that a parametric analysis is capable of producing a range of solutions that vary across decision, objective, and meta-objective space. The model presented in this paper can thus be used as a tool to generate solutions that will enhance the chances of a consensus between multiple stakeholders occurring. In the particular example in Section 4, this occurred with relatively high levels of centralisation, low levels of non-compensation between objectives, and high levels of non-compensation. The prime usage of the model is in a prescriptive sense to produce implementable solutions to complex multi stakeholder multi-objective decision network problems. It can, however, also be used in a more descriptive sense to simulate the effects of different levels of centralisation, 
non-compensation between objectives and non-compensation between stakeholders on the solutions generated in decision, objective and meta-objective space.

This paper has presented the model as a complete technique, and it can be used as such. However, it also retains the flexibility associated in goal programming described in Jones and Tamiz (2010). This includes the ability to incorporate different underlying philosophies and to combine with other techniques from the fields of Operational Research and/or artificial intelligence to enhance decision making capability.

\section{Acknowledgments}

The authors wish to gratefully acknowledge financial support from grants: 2014/04353-8, 2014/01604-0 and 2015/07293-9, São Paulo Research Foundation (FAPESP), for their funding of the first author's research visit to UNESP, Brazil in 2014 where the research presented in this paper was primarily developed. The authors also wish to thank the three anonymous referees whose comments helped shape the final version of this paper.

\section{References}

Chang, C. (2008). Revised multi-choice goal programming. Applied Mathematical Modelling, 32(12), 2587-2595.

Charnes, A., \& Cooper, W. (1961). Management models and industrial applications of linear programming. New York: Wiley \& Sons.

Charnes, A., Haynes, K. E., Hazleton, J. E., \& Ryan, M. J. (1975). A hierarchical goal-programming approach to environmental land use management. Geographical Analysis, 7(2), 121-130.

Flavell, R. (1976). A new goal programming formulation. Omega, 4(6), 731-732.

Gebrezgabher, S. A., Meuwissen, M. P., \& Oude Lansink, A. G. (2014). A multiple criteria decision making approach to manure management systems in the Netherlands. European Journal of Operational Research, 232(3), 643-653.
Giménez, J. C., Bertomeu, M., Diaz-Balteiro, L., \& Romero, C. (2013). Optimal harvest scheduling in Eucalyptus plantations under a sustainability perspective. Forest Ecology and Management, 291, 367-376.

Ignizio, J. P. (2004). Optimal maintenance headcount allocation: An application of Chebyshev Goal Programming. International Journal of Production Research, 42(1), 201-210.

Jones, D. (2011). A practical weight sensitivity algorithm for goal and multiple objective programming. European Journal of Operational Research, 213(1), 238-245.

Jones, D., \& Jimenez, M. (2013). Incorporating additional meta-objectives into the extended lexicographic goal programming framework. European Journal of Operational Research, 227(2), 343-349.

Jones, D., \& Tamiz, M. (2010). Practical Goal programming. New York: Springer.

Li, C., Liang, M., Wang, Y., \& Dong, Y. (2011). Vibration suppression using two-terminal flywheel. Part II: Application to vehicle passive suspension. Journal of Vibration and Control, 18(9), 1353-1365.

Li, X., Beullens, P., Jones, D., \& Tamiz, M. (2008). An integrated queuing and multi-objective bed allocation model with application to a hospital in China. J Oper Res Soc, 60(3), 330-338.

Nixon, J., Dey, P., Davies, P., Sagi, S., \& Berry, R. (2014). Supply chain optimisation of pyrolysis plant deployment using goal programming. Energy, 68, 262-271.

Pareto, V. (1896). Cours d'économie politique. Lausanne: F. Rouge.

Rawls, J. (1973). A theory of justice. Oxford: Oxford University Press.

Romero, C. (2001). Extended lexicographic goal programming: A unifying approach. Omega, 29(1), 63-71.

Romero, C. (2004). A general structure of achievement function for a goal programming model. European Journal of Operational Research, 153(3), 675-686.

Romero, C., Tamiz, M., \& Jones, D. F. (1998). Goal programming, compromise programming and reference point method formulations: Linkages and utility interpretations. Journal of the Operational Research Society, 49(9), 986-991.

Simon, H. (1957). Models of man. New York: Wiley \& Sons.

Tamiz, M., Azmi, R. A., \& Jones, D. F. (2013). On selecting portfolio of international mutual funds using goal programming with extended factors. European Journal of Operational Research, 226(3), 560-576.

Rodríguez Uría, M., Caballero, R., Ruiz, F., \& Romero, C. (2002). Meta-goal programming. European Journal of Operational Research, 136(2), 422-429.

Wang, Z., \& Li, K. W. (2015). A multi-step goal programming approach for group decision making with incomplete interval additive reciprocal comparison matrices. European Journal of Operational Research, 242(3), 890-900. 